\title{
Accesibilidad y equidad espacial al transporte público para pacientes con enfermedad neurodegenerativa en Santiago de Cali, Colombia
}

\author{
Public transport accessibility and spatial equity for patients \\ with neurodegenerative disease in Santiago de Cali, Colombia
}

\author{
Camila Andrea Wilches Astudillo ${ }^{1}$ \\ Ciro Jaramillo ${ }^{2}$ \\ Jackeline Murillo-Hoyos $^{3^{*}}$
}

\section{Resumen}

Los sistemas de transporte público suelen ser en ciertos casos el único medio disponible para que algunos grupos de población accedan a servicios que cubren sus necesidades básicas y esenciales. Sin embargo, los diseños de estos sistemas no garantizan una accesibilidad adecuada y funcional, en especial para la población con algún tipo de discapacidad, disminuyendo la calidad de vida de estos usuarios y convirtiéndose en un factor de exclusión social. En este contexto, empleando herramientas de análisis espacial se evaluó la equidad espacial de las condiciones de acceso al sistema de transporte público para la población con enfermedad neurodegenerativa en la ciudad de Santiago de Cali, Colombia. Se estimó la equidad espacial a través de un valor único empleando la curva de Lorenz y el coeficiente de Gini. Esta evaluación facilita el seguimiento a la provisión de servicios generando insumos para establecer estrategias de planificación y operación del sistema de transporte. Se encontró un considerable grado de desigualdad para la provisión de transporte público, debido a múltiples factores, como la disponibilidad de caminata de los pacientes y la proximidad entre la residencia del paciente a una parada y/o un corredor vial del sistema de transporte.

Palabras clave: Accesibilidad; Equidad espacial; Enfermedad de Parkinson, Indicador provisión de transporte; Coeficiente de Gini; Curva de Lorenz; Santiago de Cali, Colombia.

\footnotetext{
Abstract

Public transport systems are in some cases the only available option for a population group to access services that meet their basic and essential needs. However, the system's design does not guarantee appropriate and functional accessibility, especially for those users with some type of disability. This reduces the quality of life of these users and becomes a factor of social exclusion. The spatial equity of the conditions of access to the public transport system for the population with neurodegenerative disease in the city of Santiago de Cali, Colombia, was evaluated using spatial analysis tools. Spatial

1 Programa Académico Ingeniería Topográfica. Universidad del Valle, Colombia. camila.wilches@correounivalle.edu.co

2 Escuela de Ingeniería Civil y Geomática, Facultad de Ingeniería, Universidad del Valle, Colombia. ciro.jaramillo@correounivalle.edu.co

3 Escuela de Ingeniería Civil y Geomática, Facultad de Ingeniería, Universidad del Valle, Colombia. jackeline.murillo@correounivalle. edu.co. * Autora para correspondencia 
equity was estimated through a single value using the Lorenz curve and Gini coefficient. This evaluation facilitates the monitoring of service provision by generating inputs to establish planning and operation strategies for the transportation system. A considerable degree of inequality was found in the provision of public transport, due to multiple factors, such as ability to walk and the proximity between the patient's residence and a bus-stop and/or a road corridor of the transport system.

Keywords: Accessibility; spatial equity; Parkinson's disease, provision of transport indicator; Gini coefficient; Lorenz curve; Santiago de Cali, Colombia.

\section{Introducción}

Los procesos de planificación urbana se han orientado hacia una política de movilidad que prioriza el uso de los sistemas integrados de transporte público, como medida de acceso equitativo a todos los usuarios (Delmelle \& Casas, 2012; Camporeale, Caggiani \& Ottomanelli, 2019; Delbosc \& Currie, 2011; Ruiz, Pons, Lladó \& Reynés, 2016; Welch \& Mishra, 2013). En Colombia la implementación y puesta en marcha de este tipo de sistemas en los últimos años, ha sido el auge principal de las ciudades capitales, dado el aumento poblacional y la demanda que surge de la necesidad de transportarse de un lugar a otro. Sin embargo, pese la implementación de políticas orientadas a mejorar los sistemas de transporte, es evidente que aún existen deficiencias a nivel de infraestructura, calidad del servicio e inclusión social (Jaramillo, Lizárraga \& Grindlay, 2012; Delmelle \& Casas, 2012). Diversos autores sostienen que la exclusión social es uno de los puntos claves para entender el acceso a los sistemas de transporte y que este depende de la distribución espacial de la población, la infraestructura, el servicio de transporte y la condición socioeconómica de la persona (Martínez, 2012; Ruiz, Pons \& Lladó, 2017). De manera que esta falta de acceso ocasiona procesos de exclusión social a ciertos grupos de población, sobre todo aquellos considerados como vulnerables (Farrington, 2007; Keeling, 2008; Vasconcellos, 2001).

Es conveniente reconocer que la actividad del transporte en el ámbito urbano se asocia al término de movilidad por enfatizar el desplazamiento de personas en esta escala territorial. El aumento en las actividades y la demanda de servicios de los individuos han obligado no solo a mejorar los sistemas de transporte, sino a generar políticas de movilidad (Currie, 2010; Delmelle \& Casas, 2012; Litman, 2005; Ramjerdi, 2006). En ese sentido, la movilidad surge por la interacción entre las actividades a desarrollar por parte de los usuarios y el uso del suelo, determinando estos requerimientos desde la perspectiva de accesibilidad y equidad espacial (Church, Frost \& Sullivan, 2000; Delbosc \& Currie, 2011; Lucas, 2012; Pereira, Schwanen \& Banister, 2017).

La accesibilidad es un elemento clave en la formulación de políticas de transporte, planificación urbana y salud pública (Salze et al., 2011) al establecer una relación en términos de movilidad, donde la posibilidad de trasladarse de un lugar a otro representa una necesidad básica del individuo y una herramienta útil en la implementación de políticas gubernamentales. La falta de accesibilidad a los sistemas de transporte público se considera un factor determinante en términos de exclusión social, en el que la inequidad al acceso y a los derechos sociales de personas con discapacidad física y adultos mayores se ven perjudicados (Casas, 2007; Jaramillo et al., 2012; Welch \& Mishra, 2013).

La accesibilidad se considera un componente clave del transporte y se define como la capacidad que tiene un individuo para llegar a diferentes lugares o de un lugar ser alcanzado. Es un indicador de la estructura espacial subyacente, ya que tiene en cuenta la ubicación y la desigualdad asociada a la separación entre el origen y el destino de un viaje. La accesibilidad se basa en dos aspectos fundamentales: el primero, asociado a la localización de los lugares con relación a la oferta de transporte; y el segundo, se refiere a la distancia que se deriva de la conectividad entre lugares (Geurs \& van Wee, 2004). Dicha conectividad sólo puede existir cuando hay una posibilidad de vincular dos lugares a través de la oferta de transporte (Rodrigue, Comtois \& Slack, 2016). La accesibilidad se estima a través de indicadores que miden la capacidad de un lugar para ser alcanzado o para alcanzar diferentes lugares que se conectan mediante un sistema de transporte (Kondo, Barber \& Sherman, 1974). La accesibilidad considera todos los modos de transporte. Su evaluación se enfoca en parámetros operacionales de la oferta asociada al servicio del sistema de transporte (horarios, frecuencia, capacidad, rutas, infraestructura, etc.) y los usos del suelo (densidad de oportunidades y diversidad de actividades) (Geurs \& van Wee, 2004; Lovett, Haynes, Súnnenberg \& Gale, 2002; Monzón, 1988; Talavera \& Valenzuela, 2014). 
Existe una variedad de medidas de accesibilidad, y pueden estar basadas en la localización, en el individuo, en la utilidad, en la infraestructura, medidas de separación espacial o de redes (Geurs \& van Wee, 2004; Talavera \& Valenzuela, 2014). En el contexto de esta investigación, se consideraron las medidas basadas en la localización tanto de los pacientes, como de las estaciones de parada del Sistema Integrado de Transporte Masivo (SITM), empleando un indicador que evalúa la provisión de transporte público desde la perspectiva de la población objeto de estudio, los pacientes con enfermedad de Parkinson. La accesibilidad en el contexto de la atención primaria de los servicios de salud es reconocida como un impulso importante de la salud general de la población (Merciu, Stoian, Merciu \& Saghin, 2013).

Desde la teoría de la justicia espacial, el espacio geográfico concebido como un bien público al servicio de la justicia, requiere una organización que permita el acceso equitativo de todos los ciudadanos a las libertades fundamentales, en particular en materia de salud y educación, planteando enormes desafíos para lograr la equidad espacial (Lévy, Fauchille \& Póvoas, 2018). Los conceptos de equidad, justicia, e igualdad espacial se presentan en la literatura estrechamente conectados y con dificultad de ser desvinculados (Moreno, 2006). La justicia ideal convoca a la eliminación de todas las desigualdades, que revisadas desde la perspectiva del componente espacial representaría igualar las oportunidades de los ciudadanos y su acceso a esas oportunidades. Se entiende por equidad el tratamiento imparcial que tienen todas las personas independientemente de su posición y origen social. Equidad señala ausencia de diferencias evitables, injustas o remediables entre grupos de personas definidas social, económica, demográfica o geográficamente.

La equidad está relacionada con las oportunidades de acceso al bienestar social y a la distribución de beneficios y de costos del desarrollo, a través del establecimiento de reglas que aseguren dicha imparcialidad (Espina Prieto \& Varela., 2010; Ministerio de Salud y Protección Social, 2015). El principio de equidad espacial refiere a la distribución geográfica de un equipamiento, aludiendo directamente al concepto de igualdad, que es la medida que mejor valora las distribuciones. De manera que, la equidad espacial depende de la facilidad de acceso y de la variabilidad de las distancias, tiempos y costos que separan a cada individuo de los servicios o equipamientos más próximos (Rojas \& Martínez Bascuñán, 2014).

Para reconocer la aplicación de los conceptos de equidad, es fundamental reconocer los conceptos de equidad horizontal y vertical planteados por Truelove (1993). En el contexto de igualdad todas las personas tienen derechos y obligaciones idénticas, es decir, individuos en similares circunstancias, deben ser tratados de la misma manera, esto ha sido denominado equidad horizontal, y todos los ciudadanos reciben los mismos beneficios y cargas (Litman, 2002; Camporeale et al., 2019; Ruiz et al., 2016; Ruiz et al., 2017). En el caso contrario, cuando circunstancias personales difieren entre los individuos, es necesario revisar el reparto de utilidades y desutilidades para una partición de forma justa, imparcial y equitativa, denominado equidad vertical. En el caso de este estudio, la población objeto de análisis tiene circunstancias personales que difieren, por lo que el análisis se centra en términos de equidad vertical (Litman, 2002; Camporeale et al., 2019; Delbosc \& Currie, 2011; Ruiz et al., 2016).

La equidad supone la generación de oportunidades de acceso y control al transporte público seguro, conectando todos los espacios de la ciudad y teniendo en cuenta necesidades heterogéneas de los usuarios. El primer paso para entender el problema del transporte como un asunto de inequidad, radica no solo en tener en cuenta los aspectos técnicos de la accesibilidad, sino en involucrar una serie de recursos comunes y de uso público en beneficio de un grupo de personas (Alatorre, 2004; Mesa \& Machado, 2013). El establecimiento de métodos capaces de determinar niveles de equidad territorial junto con los de eficiencia que acompañen el bienestar humano, desde los procesos de diagnóstico para orientar las soluciones a problemas con impacto en la construcción de políticas públicas, ha sido objeto de revisión ampliamente en la literatura (Moreno, 2006).

Desde la perspectiva de salud pública, se han realizado estudios que evalúan la accesibilidad a servicios relacionados con la salud pública (Guagliardo, 2016; Huerta Munoz \& Källestål, 2012; Luo \& Wang, 2003; Moreno, García-Alonso, Negrín Hernández, Torres-González \& Salvador-Carulla, 2008) orientados en su mayoría a evaluar las barreras de accesibilidad que más se presentan para los pacientes (Asprilla, 2014; Gene \& Berrocal, 2017; Goyes \& Díaz, 2015). Conocer la distribución geográfica de pacientes con enfermedad motora es una información útil para la planificación de los servicios de transporte y de salud.

Los sistemas de transporte público suelen ser, en ciertos casos el único medio disponible que tienen ciertos grupos de población para acceder a los servicios que cubren sus necesidades básicas y esenciales. Los diseños de los sistemas de transporte público en las principales ciudades de Colombia no son garantía 
de una accesibilidad adecuada y funcional, en especial para la población que padece algún tipo de discapacidad, lo que genera una disminución paulatina en la calidad de vida de estos usuarios y sus familias, convirtiéndose en un factor determinante en términos de exclusión social (Cebollada \& Avellaneda, 2008; Guzmán, Oviedo \& Rivera, 2017; Lazo Corvalán, 2008; Martínez, 2012; Rodriguez \& Targa, 2004).

La enfermedad de Parkinson es un trastorno neurodegenerativo crónico de inicio insidioso, caracterizado por la presencia de sintomatología predominante motora que afecta a nivel mundial y tiene una tasa de incidencia actual de 4,5 a 19 personas por cada 100.000 habitantes por año. Es una enfermedad progresiva, sin cura, que disminuye la capacidad motora y afecta las actividades diarias de las personas que la padecen. La enfermedad de Parkinson, al igual que en cualquier otro trastorno crónico discapacitante, tiene en cuenta tres perspectivas: la del paciente, quien padece la enfermedad y está sujeto a la evolución del cuadro clínico; la de la familia, quien en su mayoría se hace cargo de los cuidados del paciente y asume la demanda del tratamiento; y la de la comunidad, quien le corresponde el impacto de la responsabilidad social, cultural y económica. Según la Organización Mundial de la Salud (OMS), los costos del tratamiento están directamente relacionados con la accesibilidad de los pacientes a los servicios médicos y varía dependiendo de la prioridad en salud que tenga cada país (World Health Organization [WHO], 2006).

En Colombia, de acuerdo con el estudio realizado por la Unidad de Neurociencias de la Fundación Valle del Lili, hay una incidencia para enfermedad de Parkinson de 4,7 por cada 1.000 habitantes, siendo más alta que en otros países (Arboleda, Vargas \& Galicia, 2009). Se estima que más de 220.000 pacientes en el país estarían seriamente afectados con la enfermedad. En el departamento del Valle del Cauca, cerca de 5.000 personas padecen la enfermedad, de los cuales 2.000 pacientes aproximadamente, residen en el municipio de Santiago de Cali y cerca de 480 pacientes se encuentran afiliados a la Fundación Parkinson de Colombia con sede en Santiago de Cali (Fundación Parkinson de Colombia, 2014).

La Fundación Parkinson de Colombia, es una entidad sin ánimo de lucro que surge en el año 2004, como la primera institución encargada de realizar un enfoque integral de los síntomas motores y no motores de la enfermedad de Parkinson, a través de la rehabilitación y el tratamiento oportuno. En su única sede en Santiago de Cali, la fundación atiende cerca de 480 pacientes (Fundación Parkinson de Colombia, 2014). Sobre esta población surge el interés de identificar sus condiciones de accesibilidad al SITM (con tipología de Buses de Transito Rápido - BRT-), el cual, en algunos casos, representa el único medio de transporte que tiene el paciente para acceder a la atención y tratamiento oportuno. Para evaluar la distribución de accesibilidad al SITM de los pacientes que asisten a la Fundación Parkinson sede Santiago de Cali, se emplearon herramientas de análisis espacial con el propósito de determinar el grado de equidad correspondiente.

\section{Metodología}

Para realizar la evaluación de las condiciones de accesibilidad, fue necesario reconocer la oferta del servicio de transporte provista a partir de las estaciones de parada del SITM y la localización georreferenciada de la residencia de cada uno de los pacientes (lugar donde se origina el viaje), e identificar la distancia de caminata necesaria para acceder a una estación de parada desde el lugar de residencia de los pacientes.

\subsection{Evaluación de la Provisión de Transporte}

Para evaluar el acceso al sistema de transporte público fue necesario definir un indicador que tuviera en cuenta dos elementos: el lugar de residencia del paciente $u$ origen y las estaciones de parada del SITM o destinos. Se evaluó la accesibilidad como la provisión de transporte público para cada paciente a través de un indicador topológico que considera la infraestructura, el nivel de la oferta de servicio del sistema de transporte, y está basado en el Indicador de Provisión de Transporte Público — IPTP_ (Jaramillo et al., 2012; Kondo et al., 1974; Rodrigue et al., 2016). A diferencia del índice de provisión de transporte propuesto por Currie (2010) y utilizado posteriormente por Amoroso, Salvo \& Zito (2010), que se basa en las características del área de influencia y el nivel de servicio disponible en cada parada; en el indicador de provisión de transporte público propuesto se incorporó el número de pasajeros que cada parada es capaz de absorber, en términos absolutos. 
Teniendo en cuenta la ecuación propuesta por Jaramillo et. al. (2012) en la cual se define el índice de provisión de transporte público para la ciudad de Santiago de Cali, se procede a aplicar los principios básicos planteados, efectuando modificaciones para el caso de estudio, ver Ecuación (1):

$$
I P T P I=\sum_{i=1}^{n} S_{i p} W_{c i} W_{f i}
$$

donde,

IPTPI = Índice de Provisión de Transporte Público Individualizado por paciente.

$n=$ Número de servicios del SITM (troncal, pretroncal, y alimentador).

$S_{i p}=$ Número de paradas para el servicio por paciente, según área de influencia (buffer) de caminata.

$W_{c i}=$ Factor de ponderación por la capacidad del servicio $i$.

$W_{f i}=$ Factor de ponderación por la frecuencia del servicio $i$.

En primera instancia, se estableció el IPTPI por paciente (usuario de los servicios del SITM), con un buffer de caminata de radio de $400 \mathrm{~m}$ a partir de su lugar de residencia, distancia que una persona en condiciones normales está dispuesta a recorrer para acceder a una parada de servicio de transporte público (Ammons, 2012; Demetsky \& Lin,1982; Murray \& Wu, 2003; Untermann \& Lewicki, 1984). Posteriormente, se estableció un segundo buffer de caminata con radio de $200 \mathrm{~m}$, considerando las limitaciones referidas con la caminata para un adulto mayor de 65 años (Petersen, 1968; Neilson \& Fowler, 1972), la velocidad promedio de caminata de un adulto mayor (Coffin \& Morrall, 1995), y la velocidad promedio de caminata para una persona con enfermedad de Parkinson (Paker et al., 2015). Los valores de ponderación para capacidad y frecuencia del vehículo del SITM, se definen en las Ecuaciones (2) y (3), respectivamente:

$$
W_{c i}=\frac{C_{i}}{C_{\min }}
$$

donde,

$W_{c i}=$ Factor de ponderación por la capacidad del servicio $i$.

$C_{i}=$ Capacidad del vehículo para el servicio $i$.

$C_{\min }=$ Capacidad mínima del vehículo para todos los servicios disponibles.

donde,

$$
W_{f i}=\frac{F_{i}}{F_{\min }}
$$

$W_{f i}=$ Factor de ponderación por la frecuencia del servicio $i$.

$F_{i}=$ Frecuencia para el servicio $i$.

$F_{\text {min }}=$ Frecuencia mínima para todos los servicios disponibles.

La red de corredores troncales se integra con las redes de corredores pretroncales y las redes de corredores complementarios operados por buses de menor capacidad (Figura 1) (Metro Cali, 2016). Las características disponibles para el servicio de buses fueron calculadas a partir de datos consolidados de la oferta del SITM (Tabla 1), el cual conecta la ciudad a través de un conjunto de corredores troncales con carriles segregados y preferenciales, destinados en forma exclusiva a la operación de autobuses de alta —articulados-y mediana capacidad — padrones-.

Tabla 1. Características por Tipo de Corredor servicios del SITM

\begin{tabular}{lccc}
\hline \multicolumn{1}{c}{ Tipo de Corredor } & $\begin{array}{c}\mathbf{N}^{\mathbf{o}} \text { Total } \\
\text { Paradas }\end{array}$ & $\begin{array}{c}\text { Capacidad del Vehículo } \\
\text { (Pasajeros), } \boldsymbol{C}_{\boldsymbol{i}}\end{array}$ & $\begin{array}{c}\text { Frecuencia Promedio } \\
\text { (veh/h), } \boldsymbol{F}_{\boldsymbol{i}}\end{array}$ \\
\hline Troncal & 139 & 160 & 7,6 \\
Pretroncal & 979 & 80 & 6,3 \\
Alimentador & 968 & 60 & 5,7 \\
\hline
\end{tabular}

Elaboración propia 


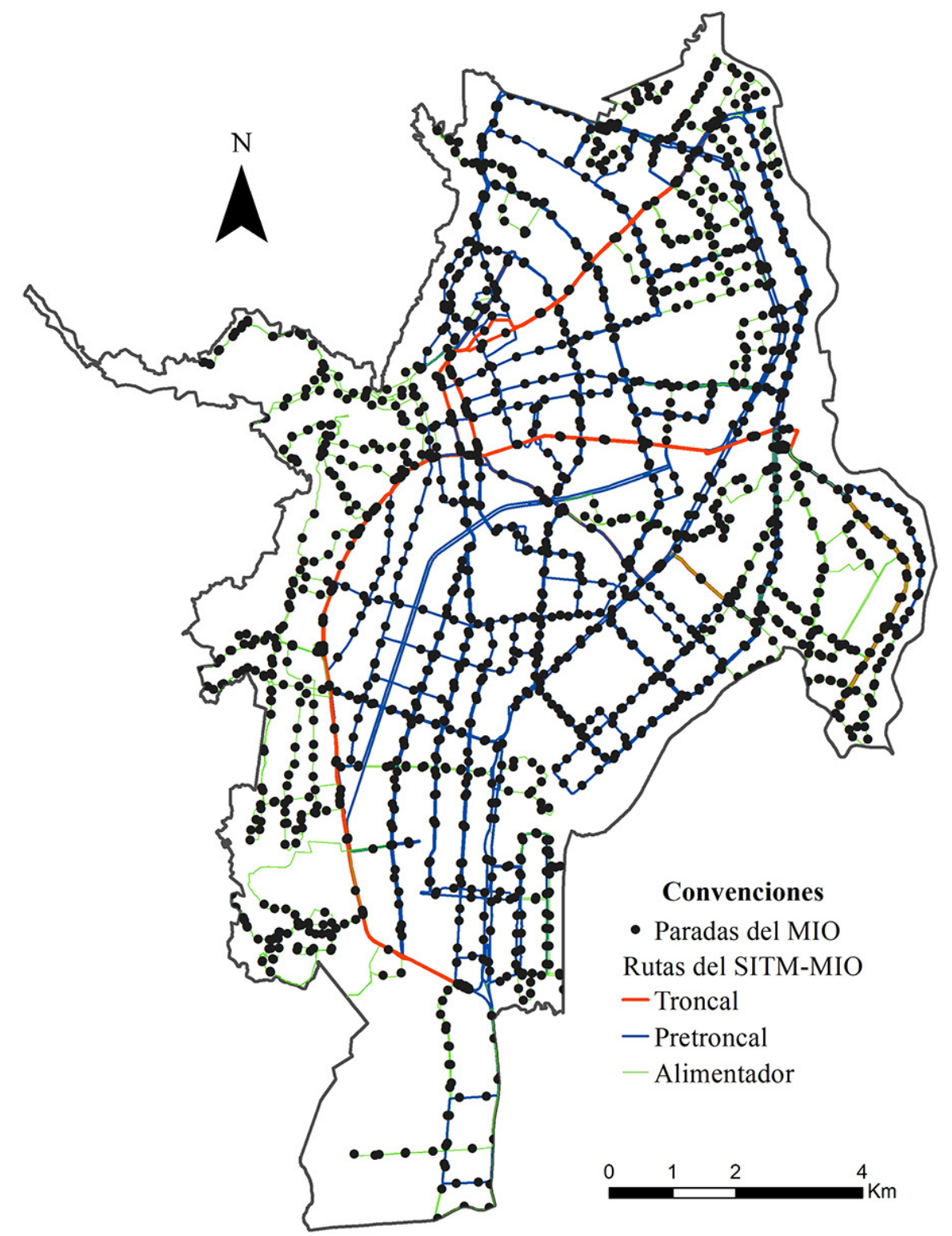

Elaboración propia

A cada registro de la base de datos de pacientes con enfermedad de Parkinson atendidos en la Fundación Parkinson de Colombia en la sede Santiago de Cali, se le definió un atributo código "Id", correspondiente al número único de identificación de cada paciente, obteniendo un total de 363 registros. Los registros suministrados se encontraban anonimizados, sin descripción alguna que comprometiera información personal de los pacientes. La población empleada representa una muestra de aproximadamente el 20\% de la población registrada con enfermedad de Parkinson en la ciudad. A partir de procesos de geocodificación y georreferenciación de cada uno de los registros del lugar de residencia de los pacientes, se construyó un mapa de densidad de Kernel empleando un radio superficial de 1000 m (Figura 2) con el propósito de reconocer la distribución espacial de la población objeto de estudio.

Posterior al cálculo del IPTPI para cada paciente registrado en la base de datos y para cada buffer de caminata desde su lugar de residencia ( $400 \mathrm{~m}$ y $200 \mathrm{~m}$ ), se realizó el respectivo análisis estadístico y estimación de ojivas de frecuencia acumulada. Finalmente, con el propósito de reconocer la distribución espacial del IPTPI se empleó el método de interpolación inverse distance weighting (IDW), conservando los parámetros establecidos por defecto del software ArcGIS ${ }^{\circ}$. 
Figura 2. Distribución espacial lugar de residencia pacientes atendidos en la Fundación Parkinson de Colombia, sede Santiago de Cali

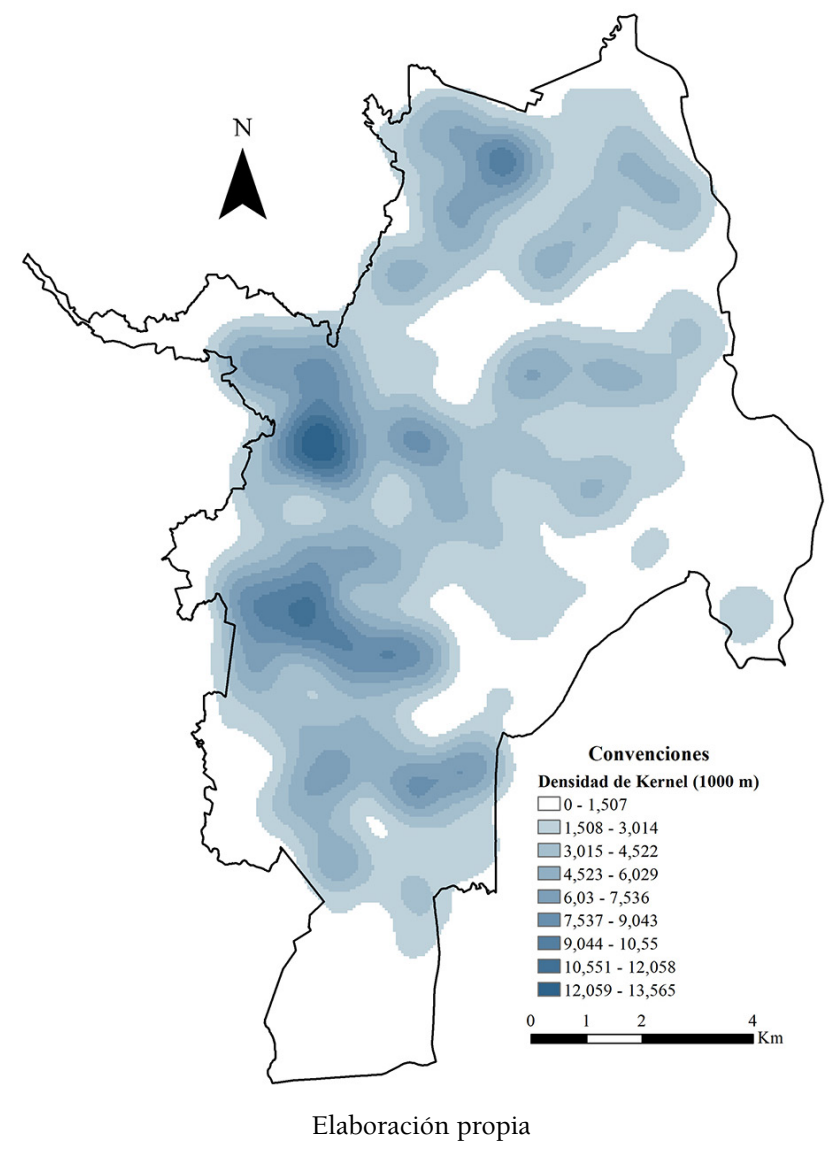

\subsection{Evaluación de la Equidad Espacial}

Para evaluar la equidad espacial se empleó la curva de Lorenz y el coeficiente de Gini (Gini, 1912; Lorenz, 1905). Con la Curva de Lorenz se representó gráficamente la distribución del IPTPI con respecto al número de pacientes con trastorno degenerativo. La curva se graficó considerando en el eje horizontal el porcentaje acumulado de pacientes con trastorno degenerativo y en el eje vertical el porcentaje acumulado de provisión de servicio de transporte público. El coeficiente de Gini representa el área de concentración entre la curva de Lorenz y la línea de igualdad perfecta, ya que expresa una proporción del área encerrada por el triángulo definido por la línea diagonal de igualdad perfecta y la línea curva de desigualdad perfecta. Cuanto más cercano sea el coeficiente a 1, más desigual será la distribución. Para ello, se tuvo en cuenta la Ecuación (4) según Rodrigue et al. (2016).

$$
G=1-\sum_{i=0}^{N}\left(\sigma Y_{i}+\sigma Y_{i-1}\right)\left(\sigma X_{i}-\sigma X_{i-1}\right)
$$

donde,

$G=$ Valor del índice de Gini.

$\sigma Y_{i=}$ Proporción real acumulada de la población dotada de provisión de transporte.

$\sigma X_{i}$ Proporción ideal acumulada de la población dotada de provisión de transporte.

$\mathrm{N}=$ Número de elementos.

\section{Resultados}

A continuación, se presentan los productos asociados al desarrollo metodológico descrito en la sección previa. Los resultados obtenidos en este análisis son representativos para aproximadamente el 20\% de la población registrada con enfermedad de Parkinson en la ciudad de Santiago de Cali. 


\subsection{Evaluación de la Provisión de Transporte Público}

En la Tabla 2 se presentan los estadísticos del IPTPI para los dos buffers de caminata planteados, 400 y $200 \mathrm{~m}$. El comportamiento del indicador IPTPI de $400 \mathrm{~m}$ revela que el valor máximo es un caso atípico que refiere a la existencia de intervalos vacíos entre 65 y 113,80, lo que explica bajos números tanto para la media como para la mediana y un elevado coeficiente de variación. Para el IPTPI de 200 m el rango de valores es mucho menor respecto al IPTPI de $400 \mathrm{~m}$, debido a la disminución en el número de paradas disponibles en el radio de búsqueda. Ambos radios de búsqueda presentan una asimetría positiva y una distribución leptocúrtica.

Tabla 2. Resumen estadístico del para cada buffer de caminata

\begin{tabular}{cccccccccc}
\hline Indicador & Media & Mediana & Coeficiente de Variación (\%) & Desviación & Mínimo & Máximo & Asimetría & Curtosis \\
\hline $400 \mathrm{~m}$ & 16,70 & 14,20 & 84,90 & 14,20 & 0,00 & 113,80 & $2,04+$ & 9,96 \\
$200 \mathrm{~m}$ & 2,94 & 2,00 & 111,20 & 3,27 & 0,00 & 24,90 & $2,18+$ & 10,80 \\
\hline
\end{tabular}

Elaboración propia

Con relación a la frecuencia acumulada presentada en la Figura 3, se destaca que, valores del IPTPI entre 0 y 15 corresponden aproximadamente al 50\% y al 98\% de los pacientes, para los rangos de caminata de $400 \mathrm{~m}$ y $200 \mathrm{~m}$, respectivamente. El número de pacientes sin paradas aproximadamente se duplica.

Figura 3. Frecuencia Acumulada para el IPTPI

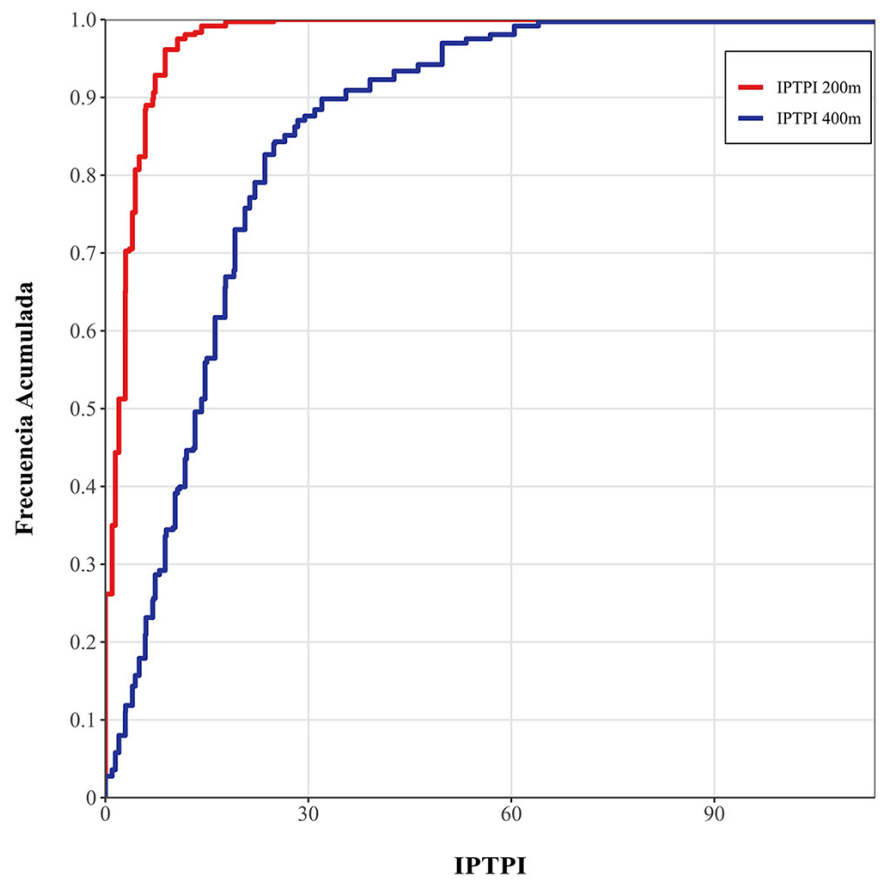

Elaboración propia

Las Figuras 4 ilustran la distribución espacial del IPTPI para los diferentes niveles de provisión de transporte público usando el método de interpolación IDW. En la Figura 4a se presenta el IPTPI 400 m, en la que se observa que las zonas de residencia de los pacientes atendidos en la Fundación Parkinson de Colombia (sede Santiago de Cali) con mejor provisión de servicio de transporte público se encuentran por encima de un valor de 30. Se evidencian zonas de gran extensión que señalan baja provisión de transporte público para los pacientes residentes en las zonas sur, centro-occidente, sur-oriental y una franja irregular del centro al oriente de la ciudad. Por otra parte, se observa una franja desde la zona centro-occidente hacia la zona norte con una provisión favorable de servicio de transporte público para los pacientes residentes en esas zonas debido a la oferta tanto de corredores como de número de paradas próximos a los lugares de residencia en el buffer de caminata. 
En la Figura 4b se presenta el IPTPI $200 \mathrm{~m}$, en la que se observa que las zonas de residencia de los pacientes atendidos en la Fundación Parkinson de Colombia (sede Santiago de Cali) con mejor provisión de servicio de transporte público se encuentran por encima de un valor de 6 . El comportamiento del IPTPI para un buffer de caminata de $200 \mathrm{~m}$, sigue la tendencia de su análogo de $400 \mathrm{~m}$, y se agudiza la reducción en la provisión de transporte, debido a que la restricción en el radio de búsqueda implica una disminución considerable en la oferta de paradas de los servicios del SITM próximas a los lugares de residencia de los usuarios con enfermedad neurodegenerativa considerados en este estudio.

En términos cualitativos el comportamiento de los valores se mantiene, mientras cuantitativamente el comportamiento tiende a ser desfavorable al reducir el buffer de caminata a la mitad para acceder a una parada del servicio de buses.

Figura 4. IPTPI para los pacientes atendidos en la Fundación Parkinson de Colombia, sede Santiago de Cali

a) IPTPI, buffer de caminata $400 \mathrm{~m}$

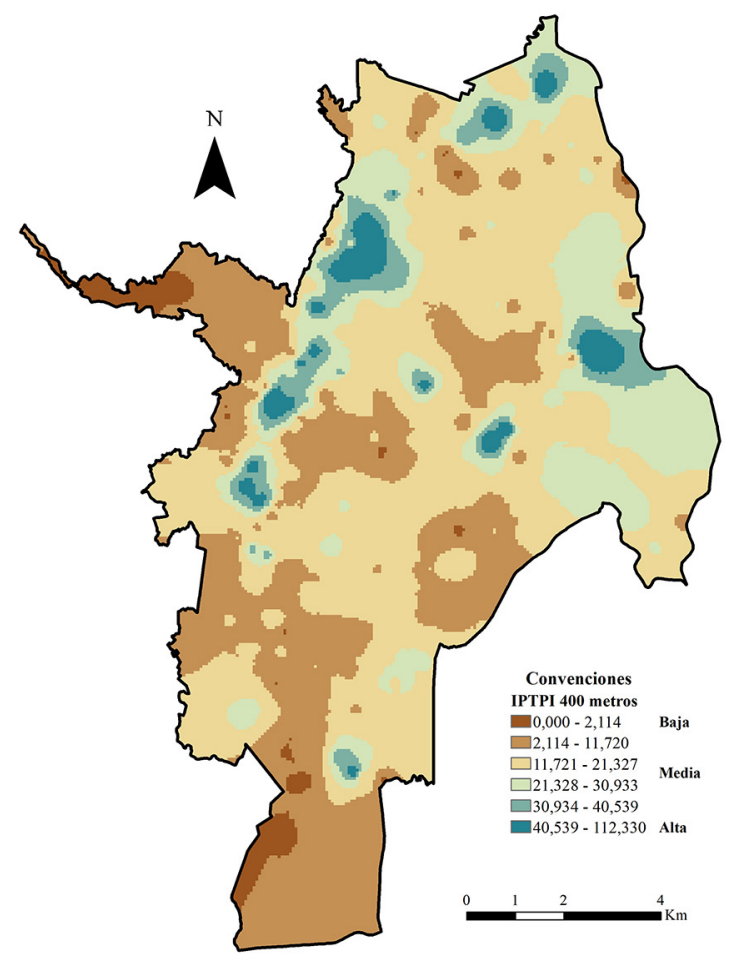

b) IPTPI, buffer de caminata $200 \mathrm{~m}$

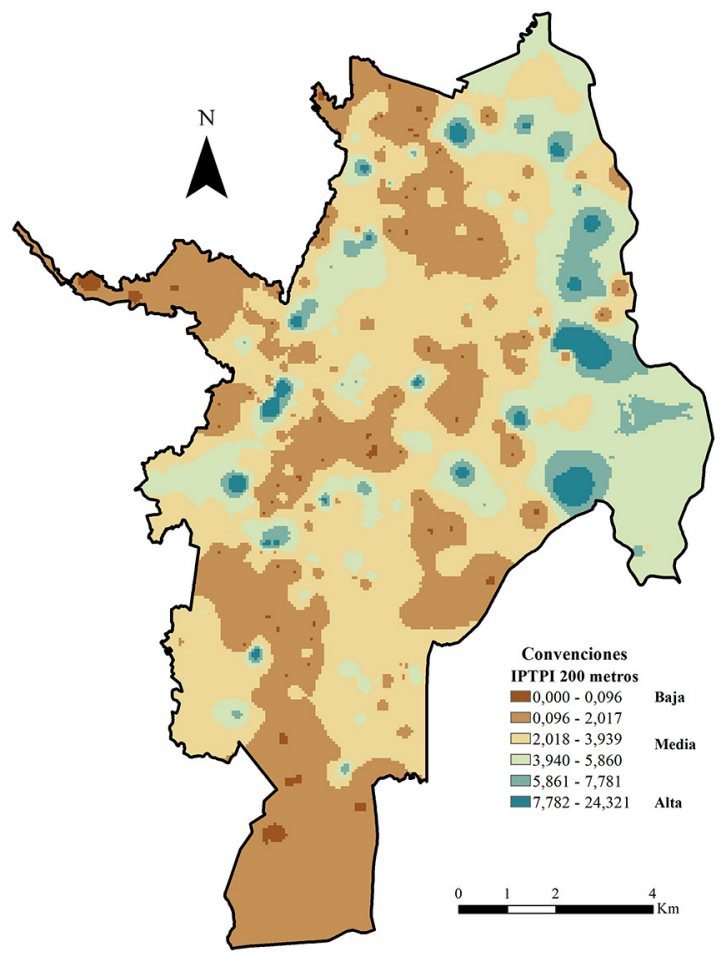

Elaboración propia

\subsection{Evaluación de la Equidad Espacial}

La Figura 5 presenta las curvas de Lorenz para el IPTPI para buffers de caminata de radio de $400 \mathrm{y}$ $200 \mathrm{~m}$. Se observa que, para el buffer de $400 \mathrm{~m}$, el $40 \%$ de los pacientes menos favorecidos tienen una provisión de servicio de transporte público inferior al 15\% y el $60 \%$ de los pacientes una provisión de servicio de transporte público ligeramente superior al 30\%. Este indicador tiene un coeficiente de Gini de 0,42 representando una inequidad resultante de la separación entre la curva y la línea de perfecta equidad. Para el buffer de caminata de radio de $200 \mathrm{~m}$, se observa que el $40 \%$ de los pacientes menos favorecidos tienen una provisión de servicio de transporte público inferior al 5\% y el $60 \%$ de los pacientes una provisión de servicio de transporte público del 20\%. Este indicador tiene un coeficiente de Gini de 0,54 representando una inequidad resultante de la amplia separación entre la curva y la línea de perfecta equidad.

Al considerar una reducción del buffer de caminata como consecuencia de las limitaciones de movilidad estimadas para pacientes con enfermedad neurológica se observa un incremento de inequidad del $29 \%$, tomando como referencia el coeficiente de Gini para el indicador de provisión de transporte público con buffer de caminata de radio de $400 \mathrm{~m}$. Desde la perspectiva de provisión de estaciones de parada del 
SITM, se observó que el $7 \%$ y el 28\% de la población objeto de estudio no tienen acceso a una parada del SITM en los buffers de caminata de $400 \mathrm{~m}$ y $200 \mathrm{~m}$, respectivamente.

Figura 5. Curva de Lorenz para el IPTPI

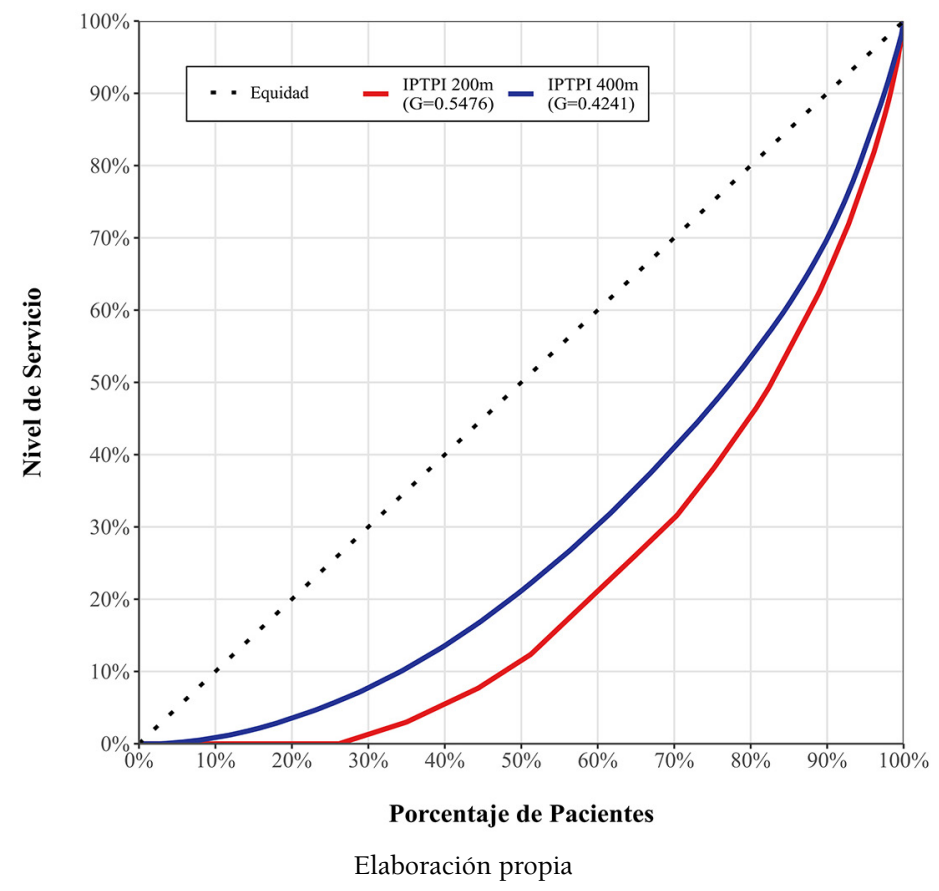

\section{Discusión de resultados}

En la evaluación de la provisión de transporte los resultados obtenidos fueron contrastados con los hallazgos obtenidos por Delmelle $\&$ Casas (2012) en cuanto a las zonas de cobertura teniendo en cuenta los tiempos de caminata para llegar a las paradas del SITM. De igual forma se aprecia coherencia con lo obtenido por Jaramillo et al. (2012) sobre la distribución espacial de la provisión de transporte del SITM en la ciudad de Cali. El estudio realizado por Delmelle \& Casas (2012) identifica que el SITM es fundamental para proporcionar acceso equitativo a los usuarios permitiendo el alcance a las oportunidades en la escala territorial de operación del sistema. Los hallazgos en dicho estudio indican una estimación conservadora del acceso en modo caminata al SITM, con la gran mayoría del área de la ciudad cubierta por al menos 20 minutos a pie, a excepción de varios vecindarios en el borde occidental de la ciudad. Este tiempo de caminata representa longitudes entre 1-1,5 $\mathrm{km}$ para personas sin compromiso de sus funciones motoras. En contraste, considerando las limitaciones de los usuarios con enfermedades neurodegenerativas, estos tendrían una cobertura estimada para estaciones de parada del SITM que se encuentren a no más de 5 minutos desde sus lugares de residencia.

Por otra parte, en el trabajo de Jaramillo et al. (2012), el SITM se reconoce como una alternativa de transporte que beneficia la movilidad urbana, sin embargo, se evidencian oportunidades de mejora asociadas con la cobertura espacial y de una mayor atención hacia los usuarios que poseen elementos característicos de las necesidades sociales del servicio de transporte, que no suelen ser considerados en los procesos de planificación de los sistemas de transporte. Esto está en sintonía con los hallazgos de este estudio con relación a los pacientes de la enfermedad neurodegenerativa. Asociado a esto, de manera análoga, Jaramillo et al. (2012), muestran diferencias espaciales entre los indicadores de provisión estimados y las necesidades de los pacientes de interés. Los resultados coinciden en indicar que el SITM puede y debe mejorar sus servicios implementando medidas específicas que tomen en cuenta las características de ciertos colectivos que hacen parte importante de las necesidades sociales del servicio de transporte en la ciudad de Santiago de Cali.

Con relación a la evaluación de la equidad espacial, los resultados obtenidos en este estudio son más críticos que lo hallado por Cardona \& Jaramillo (2020) para el transporte activo y para la población en general de la ciudad de Santiago de Cali. La condición de los pacientes con enfermedad de Parkinson es 
mucho más desfavorable desde la perspectiva del transporte motorizado, en especial el SITM disponible en la zona de estudio. En este sentido Cardona \& Jaramillo (2020) determinan en su análisis que la distribución de los indicadores de accesibilidad basados en la ciclo-infraestructura es más equitativa (coeficiente de Gini entre el 22\% y el 27\%), considerando las condiciones socioeconómicas de la población en el ámbito urbano, dependiendo de los indicadores y sectores de la población considerada. Estas medidas, en el caso de la evaluación análoga con el SITM y los pacientes objeto de este estudio es del orden de $42 \%$ y el 54\%, mostrando una situación más desbalanceada con valores cercanos al doble de los obtenidos por Cardona \& Jaramillo (2020). Los dos estudios dejan evidencias sobre la necesidad de considerar una reestructuración de la provisión de transporte que sea más cercana a la necesidad de viajes, que es distinto a la demanda satisfecha por un modo de transporte en particular.

\section{Conclusiones}

Con relación a los estadísticos del IPTPI: media, mediana y máximo para los buffers de caminata considerados, $400 \mathrm{~m}$ y $200 \mathrm{~m}$, se observa que al reducir en un 50\% la distancia del buffer de caminata para acceder a una parada del SITM, estos estadísticos se reducen en una proporción de aproximadamente 6 a 1 para la media, 7 a 1 para la mediana, y 5 a 1 para el valor máximo del indicador.

Desde la perspectiva de provisión de estaciones de parada del SITM, se observó una relación de 1 a 4 para pacientes sin acceso al SITM en los buffers de caminata de $400 \mathrm{~m}$ y $200 \mathrm{~m}$, respectivamente. Las zonas críticas en las que residen los pacientes atendidos en la Fundación Parkinson de Colombia, sede Santiago de Cali, se evidencian hacia los sectores sur, centro-occidente, sur-oriental y una franja irregular del centro al oriente de la ciudad, que señalan baja densidad de paradas sobre los corredores viales de la red.

Las zonas de residencia de los pacientes desde el sector centro-occidente hacia sector norte, evidencian una alta provisión del servicio de transporte individualizado, debido a la elevada densidad de paradas que satisfacen el acceso de los pacientes al SITM desde su lugar de residencia dentro de cada buffer de caminata considerado.

El comportamiento espacial de la provisión de transporte para el $40 \%$ de los pacientes menos favorecidos se reduce en una proporción de 3 a 1, al reducir en un 50\% la distancia del buffer de caminata (400 $\mathrm{m}$ a $200 \mathrm{~m}$ ) para acceder a una parada del SITM-MIO.

El Coeficiente de Gini estimado para IPTPI de $400 \mathrm{~m}$ y $200 \mathrm{~m}$, es 0,42 y 0,54, respectivamente. Estos valores representan una inequidad resultante de la separación entre la curva y la línea de perfecta equidad para ambos buffers de caminata. Al tomar como referencia el coeficiente de Gini para el buffer de caminata de $400 \mathrm{~m}$, se observa un incremento de inequidad del 31\% para el buffer de caminata de $200 \mathrm{~m}$.

Al estimar la equidad espacial a través del uso de los coeficientes de Gini y la curva de Lorenz se encontró un considerable grado de desigualdad para la provisión de transporte público individualizado, debido principalmente a que este indicador obedece a múltiples factores, como la disponibilidad de caminata de los pacientes y la proximidad entre la residencia del paciente a una parada y/o un corredor vial del SITM.

La metodología desarrollada es de fácil implementación dado que requiere solamente la articulación entre la información asociada a la operación del sistema como corredores, paradas y capacidad de los buses y la localización de la residencia de los pacientes. Integrando dicha información a través de un valor único basado en la curva de Lorenz y el coeficiente de Gini es posible medir la equidad en la provisión del servicio de transporte público para un grupo de población específico como es el caso de los pacientes con enfermedad de Parkinson. Esta metodología permite orientar estrategias en torno a la planificación y operación del sistema de transporte al reconocer grupos de población desatendidos por este servicio, para los cuales será necesario establecer esquemas operacionales que atiendan a la demanda específica del tipo de colectivo, garantizando el derecho a la equidad en lo referente a la provisión de transporte e inclusión social.

\section{Agradecimientos}

Los autores del manuscrito agradecen la valiosa colaboración de la Fundación Parkinson de Colombia en su sede de Santiago de Cali, por el suministro de las bases de datos anonimizadas de las direcciones de residencia de sus pacientes, las cuales fueron fundamentales en el desarrollo de esta investigación. 


\section{Referencias}

Alatorre, C. (2004). Inequidad social y transporte urbano: La saturación de un recurso de uso común. En X Conferencia Bienal de la Asociación Internacional para el Estudio de la Propiedad Colectiva (IASCP) "Los recursos de uso común en una era de transición global: retos, riesgos y oportunidades". Oaxaca, México. Recuperado de http://hdl.handle.net/10535/120

Ammons, D. (2012). Municipal Benchmarks: Assessing Local Performance and Establishing Community Standards. http://dx.doi.org/10.4135/9781452233468

Amoroso, S., Salvo, G., \& Zito, P. (2011). L'accessibilità dei Trasporti Pubblici come Strumento per Vincere L'esclusione Sociale. En XII Conferencia de la Sociedad Italiana de Economía del Transporte y Logística. Conferencia llevada a cabo en Roma, Italia. Recuperado de http://hdl.handle.net/10447/53639

Arboleda, A.F., Vargas, D.J., \& Galicia, J.C. (2009). Caracterización del nivel de funcionalidad cotidiana en un grupo de adultos mayores (AÑORANZAS) de COOEDUCAR-COMCAJA (Tesis de pregrado). Universidad Tecnológica de Pereira. Pereira. Recuperado de http://repositorio.utp.edu.co/dspace/ handle/11059/980

Asprilla, J. (2014). Niveles de Accesibilidad y Cobertura de la Red de Salud Pública en la ciudad de Cali (Tesis de pregrado). Universidad del Valle. Cali. Recuperado de http://bibliotecadigital.univalle.edu. co/handle/10893/8533

Camporeale, R., Caggiani, L., \& Ottomanelli, M. (2019). Modeling horizontal and vertical equity in the public transport design problem: A case study. Transportation Research Part A: Policy and Practice, 125, 184-206. https://doi.org/10.1016/j.tra.2018.04.006

Cardona, C., \& Jaramillo, C. (2020). Midiendo la equidad de acceso a la infraestructura de bicicletas en Santiago de Cali, Colombia. En Figueroa, O., Valenzuela, L.M., \& Brasileiro, A. (Coords), Desafíos del desarrollo urbano sostenible en el transporte y la movilidad (pp. 163-187). Zinacantepec: El Colegio Mexiquense, A.C.

Casas, I. (2007). Social Exclusion and the Disabled: An Accessibility Approach. The Professional Geographer, 59(4), 463-477. https://doi.org/10.1111/j.1467-9272.2007.00635.x

Cebollada, À., \& Avellaneda, P. G. (2008). Equidad social en movilidad: reflexiones en torno a los casos de Barcelona y Lima. Scripta Nova: revista electrónica de geografía y ciencias sociales, 12. Recuperado de http://www.ub.edu/geocrit/sn/sn-270/sn-270-47.htm

Church, A., Frost, M., \& Sullivan, K. (2000). Transport and social exclusion in London. Transport Policy, 7(3), 195-205. https://doi.org/https://doi.org/10.1016/S0967-070X(00)00024-X

Coffin, A., \& Morrall, J. (1995). Walking speeds of elderly pedestrians at crosswalks. Transportation Research Record, 1487, 63-67.

Currie, G. (2010). Quantifying spatial gaps in public transport supply based on social needs. Journal of Transport Geography, 18, 31-41. https://doi.org/10.1016/j.jtrangeo.2008.12.002

Demetsky, M.J., \& Lin, BB-M. (1982). Bus Stop Location and Design. Transportation Engineering Journal of ASCE, 108(4), 313-327. https://doi.org/10.1061/TPEJAN.0000991

Delbosc, A., \& Currie, G. (2011). Using Lorenz curves to assess public transport equity. Journal of Transport Geography, 19(6), 1252-1259. https://doi.org/10.1016/j.jtrangeo.2011.02.008

Delmelle, E. C., \& Casas, I. (2012). Evaluating the spatial equity of bus rapid transit-based accessibility patterns in a developing country: The case of Cali, Colombia. Transport Policy, 20(C), 36-46. https:// doi.org/10.1016/j.tranpol.2011.12.001

Espina Prieto, M., \& Varela., C. F. (2010). Desarrollo, desigualdad y políticas sociales : acercamientos desde una perspectiva compleja. Publicaciones Acuario, Centro Félix Varela.

Farrington, J. H. (2007). The new narrative of accessibility: its potential contribution to discourses in (transport) geography. Journal of Transport Geography, 15(5), 319-330. https://doi.org/https://doi. org/10.1016/j.jtrangeo.2006.11.007

Fundación Parkinson de Colombia. (2014). Base de Datos pacientes atendidos en la Sede de Santiago de Cali. 2014. Suministrada en formato digital con reserva de publicación. 
Gene, F. H., \& Berrocal, R. G. (2017). Accesibilidad física de la población a servicios de salud pública en San Pelayo y Cereté, Córdoba, Colombia, año 2015. Perspectiva Geográfica, 22(2 SE-Artículos). https://doi.org/10.19053/01233769.7599

Geurs, K. T., \& van Wee, B. (2004). Accessibility evaluation of land-use and transport strategies: review and research directions. Journal of Transport Geography, 12(2), 127-140. https://doi.org/https://doi. org/10.1016/j.jtrangeo.2003.10.005

Gini, C. (1912). Variabilità e Mutabilità (Bologna: Tipografia di Paolo Cuppini). En: Dorfman, R. (1979). A Formula for the Gini Coefficient. The Review of Economics and Statistics, 61(1), 146-149. https://doi. org/10.2307/1924845

Goyes, D.M., \& Díaz, B. (2015). Niveles de accesibilidad a los servicios de salud de urgencias, en el departamento del Valle del Cauca (Tesis de pregrado). Universidad del Valle. Cali.

Guagliardo, M. F. (2004). Spatial accessibility of primary care: Concepts, methods and challenges. International Journal of Health Geographics, 3, 1-13. https://doi.org/10.1186/1476-072X-3-3

Guzmán, L. A., Oviedo, D. R., \& Rivera, C. (2017). Assessing equity in transport accessibility to work and study: The Bogotá region. Journal of Transport Geography, 58, 236-246. https://doi.org/10.1016/j. jtrangeo.2016.12.016

Huerta Munoz, U., \& Källestål, C. (2012). Geographical accessibility and spatial coverage modeling of the primary health care network in the Western Province of Rwanda. International Journal of Health Geographics, 11, 1-11. https://doi.org/10.1186/1476-072X-11-40

Jaramillo, C., Lizárraga, C., \& Grindlay, A. L. (2012). Spatial disparity in transport social needs and public transport provision in Santiago de Cali (Colombia). Journal of Transport Geography, 24, 340357. https://doi.org/10.1016/j.jtrangeo.2012.04.014

Keeling, D. J. (2008). Latin America's Transportation Conundrum. Journal of Latin American Geography, 7(2), 133-154. https://doi.org/10.1353/lag.0.0005

Kondo, W., Barber, B., \& Sherman, L. (1974). Method for evaluating metropolitan accessibility. Transportation Research Record, 499, 70-82. Recuperado de http://onlinepubs.trb.org/Onlinepubs/ trr/1974/499/499-006.pdf

Lazo Corvalán, A. (2008). Transporte, movilidad y exclusión: El caso de Trasantiago en Chile. Scripta Nova: Revista Electrónica de Geografía y Ciencias Sociales, 12, 41. Recuperado de http://www.ub.edu/ geocrit/sn/sn-270/sn-270-45.htm

Lévy, J., Fauchille, J.N., \& Póvoas, A. (2018). Théorie de la justice spatiale. Géographies du juste et de l'injuste. Odile Jacob. Recuperado de https://www.jssj.org/article/theorie-de-la-justice-spatialegeographies-du-juste-et-de-linjustel

Litman, T. (2002). Evaluating transportation equity. World Transport Policy \& Practice, 8(2), 50-65.

Litman, T. (2005). Evaluating Transportation Equity: Guidance for Incorporating Distributional Impacts in Transportation Planning. Victoria Transport Policy Institute, Victoria, British, 8(2), 50-65. Recuperado de http://citeseerx.ist.psu.edu/viewdoc/download?doi=10.1.1.1040.6553\&rep=repl\&type=pdf

Lorenz, M. O. (1905). Methods of Measuring the Concentration of Wealth. Publications of the American Statistical Association, 9(70), 209-219. https://doi.org/10.2307/2276207

Lovett, A., Haynes, R., Sünnenberg, G., \& Gale, S. (2002). Car travel time and accessibility by bus to general practitioner services: a study using patient registers and GIS. Social Science \& Medicine, 55(1), 97-111. https://doi.org/10.1016/S0277-9536(01)00212-X

Lucas, K. (2012). Transport and social exclusion: Where are we now?. Transport Policy, 20, 105-113. https://doi.org/10.1016/j.tranpol.2012.01.013

Luo, W., \& Wang, F. (2003). Measures of Spatial Accessibility to Health Care in a GIS Environment: Synthesis and a Case Study in the Chicago Region. Environment and Planning B: Planning and Design, 30(6), 865-884. https://doi.org/10.1068/b29120

Martínez, D.C. (2012). Estrategias para promover la accesibilidad, cobertura y calidad en el sistema de transporte público urbano para la población con discapacidad física: caso Bogotá (Tesis de Maestría). Universidad Nacional de Colombia. Bogotá. Recuperado de https://repositorio.unal.edu.co/handle/ unal/11512 
Merciu, C., Stoian, D., Merciu, G., \& Saghin, I. (2013). Using GIS for calculating the accessibility to hospitals in the city of Bucharest and its metropolitan area (Romania). Geographica Pannonica, 17(4), 106-113. https://doi.org/10.5937/GeoPan1304106M

Mesa, D., \& Machado, D. (Noviemebre de 2013). La equidad social como eje transversal en las políticas de movilidad urbana. En memorias 3er Encuentro Iberoamericano de Movilidad Urbana Sostenilble. Lima, Perú. Recuperado de http://ciudadenmovimiento.org/wp-content/uploads/2014/03/2do-PremioDayana-Mesa-Darmis-Machado.pdf

Metro Cali S.A. (2016). Base de Datos Infraestructura Operacional. Suministrada en formato digital con reserva de publicación.

Ministerio de Salud y Protección Social. (2015). Observatorio para Medición de Desigualdades y Análisis de Equidad en Salud, ODES Colombia. Recuperado de http://sia.eurosocial-ii.eu/files/docs/1452159783OC_guia_ross_odes_colombia.pdf

Monzón, A. (1988). Los indicadores de accesibilidad y su papel decisor en las inversiones en infraestructuras de transporte. Aplicaciones en la comunidad de Madrid (Tesis de doctorado). Universidad Politécnica de Madrid. Madrid. http://oa.upm.es/736/1/04198801.pdf

Moreno, B., García-Alonso, C. R., Negrín Hernández, M. A., Torres-González, F., \& Salvador-Carulla, L. (2008). Spatial analysis to identify hotspots of prevalence of schizophrenia. Social Psychiatry and Psychiatric Epidemiology, 43(10), 782-791. https://doi.org/10.1007/s00127-008-0368-3

Moreno Jiménez, A. (2006-07). En torno a los conceptos de equidad, justicia e igualdad espacial. Huellas, 11, 133-142. http://www.biblioteca.unlpam.edu.ar/pubpdf/huellas/n1la09moreno.pdf

Murray, A. T., \& Wu, X. (2003). Accessibility tradeoffs in public transit planning. Journal of Geographical Systems, 5(1), 93-107. https://doi.org/10.1007/s101090300105

Neilson, G., \& Fowler, W. (1972). Relation between transit ridership and walking distances in a lowdensity Florida retirement area. Highway Research Record, 403, 26-34.

Paker, N., Bugdayci, D., Goksenoglu, G., Demircioğlu, D. T., Kesiktas, N., \& Ince, N. (2015). Gait speed and related factors in parkinson's disease. Journal of Physical Therapy Science, 27(12), 3675-3679. https://doi.org/10.1589/jpts.27.3675

Pereira, R. H. M., Schwanen, T., \& Banister, D. (2017). Distributive justice and equity in transportation. Transport Reviews, 37(2), 170-191. https://doi.org/10.1080/01441647.2016.1257660

Petersen, S. C. (1968). Walking distances to bus stops in Washington, D.C. residential areas. Traffic Engineering, 39(3), 28-35.

Ramjerdi, F. (2006). Equity Measures and Their Performance in Transportation. Transportation Research Record, 1983(1), 67-74. https://doi.org/10.1177/0361198106198300110

Rodrigue, J.P., Comtois, C., \& Slack, B. (2016). The geography of transport systems. In The Geography of Transport Systems. https://doi.org/10.4324/9781315618159

Rodriguez, D., \& Targa, F. (2004). Value of accessibility to Bogotá's bus rapid transit system. Transport Reviews, 24, 587-610. https://doi.org/10.1080/0144164042000195081

Rojas, C., \& Martínez Bascuñán, M. (2014). Evaluación de la Accesibilidad Espacial a la red hospitalaria en el área metropolitana de Concepción. Revista Digital Del Grupo de Estudios Sobre Geografía y Análisis Espacial Con Sistemas de Información Geográfica, 6, 176-200.

Ruiz, M., Seguí Pons, J. M., Mateu Lladó, J., \& Martínez Reynés, M. R. (2016). Evaluación de la equidad del servicio de transporte público: El caso de Palma de Mallorca. Estudios Geográficos, 77(281), 619646. https://doi.org/10.3989/estgeogr.201621

Ruiz, M., Segui-Pons, J. M., \& Mateu Lladó, J. (2017). Improving Bus Service Levels and social equity through bus frequency modelling. Journal of Transport Geography, 58, 220-233. https://doi. org/10.1016/j.jtrangeo.2016.12.005

Salze, P., Banos, A., Oppert, J. M., Charreire, H., Casey, R., Simon, C., \& Weber, C. (2011). Estimating spatial accessibility to facilities on the regional scale: An extended commuting-based interaction potential model. International Journal of Health Geographics, 10, 1-16. https://doi.org/10.1186/1476072X-10-2 
Talavera, R. \& Valenzuela, L.M. (2014). Guía para el análisis de la accesibilidad espacial del transporte público: enfoque desde los sistemas de metro ligero. Proyecto INTEGRAME. Laboratorio de Planificación Ambiental. Granada. Recuperado de http://www.integrame.es/wp-content/uploads/2014/07/GM Accesl.pdf

Truelove, M. (1993). Measurement of spatial equity. Environment and Planning C: 12 Government and Policy 11, 19-34. https://doi.org/10.1068/c110019

Untermann, R. K., \& Lewicki, L. (1984). Accommodating the pedestrian: adapting towns and neighborhoods for walking and bicycling. Van Nostrand Reinhold.

Vasconcellos, E. A. (2001). Urban Transport, Environment and Equity: The Case for Developing Countries https://doi.org/10.4324/9781315071756

Welch, T. F., \& Mishra, S. (2013). A measure of equity for public transit connectivity. Journal of Transport Geography, 33, 29-41. https://doi.org/10.1016/j.jtrangeo.2013.09.007

World Health Organization (WHO). (2006). Neurological disorders : public health challenges. World Health Organization. Recuperado de https://apps.who.int/iris/handle/10665/43605 